\title{
Current status of peptic ulcer disease in Port Harcourt metropolis, Nigeria
}

\author{
Emeka Ray-Offor ${ }^{1,2}$, Kalanne Ada Opusunju ${ }^{1}$
}

1. Digestive Disease Unit, Oak Endoscopy Centre Port Harcourt.

2. Department of Surgery, University of Port Harcourt Teaching Hospital Port Harcourt, Rivers State, Nigeria.

\begin{abstract}
Background: Epidemiological studies on peptic ulcer disease (PUD) have shown a recent decrease in hospital admissions in Western countries.

Objective: This paper aimed to study the current status and risk factors of PUD in a Nigerian metropolis.

Methods: A cross-sectional study of symptomatic patients at upper gastrointestinal (GI) endoscopy diagnosed with PUD from February 2014 to September 2019 at a referral endoscopy facility in Port Harcourt, Niger delta region of Nigeria. The variables studied included demographics, symptoms and duration, blood group, chronic non-steroidal anti-inflammatory (NSAID) use, smoking, endoscopic and histology findings. Statistical analysis was performed using SPSS version 20.

Results: A total of 434 upper GI endoscopies were performed during the study period with thirty-one diagnosis of PUD made. The mean age of gastric ulcer (GU) and duodenal ulcer (DU) cases were $54.4 \pm 20.2 \mathrm{yrs}$ and $48.1 \pm 14.5 \mathrm{yrs}$ respectively $(\mathrm{p}=0.367)$. GU to DU ratio was 1.4:1. H. pylori infection, chronic NSAID use and blood group O were seen in 7(22.5\%), $8(25.8 \%)$ and $18(72.0 \%)$ respectively. Major indication in 21(67.7\%) cases was gastrointestinal bleeding.
\end{abstract}

Conclusion: There is a low diagnostic rate of PUD $(6.7 \%)$ with pre-pyloric antral gastric ulcers as most common type and multifactorial aetiology.

Keywords: Gastric ulcer; duodenal ulcer; endoscopy.

DOI: https://dx.doi.org/10.4314/ahs.v20i3.50

Cite as: Ray-Offor E, Opusunju KA. Current status of peptic ulcer disease in Port Harcourt metropolis, Nigeria. Afri Health Sci. 2020;20(3): 1446-1451. https:// dx.doi.org/10.4314/abs.v20i3.50

\section{Introduction}

Peptic ulcer is a disease of antiquity with the first medical report in the $4^{\text {th }}$ century $\mathrm{BC}^{1}$. Globally, peptic ulcers are estimated to involve $5-10 \%$ of the population. ${ }^{2}$ It is a breach in gastric or duodenal epithelium extending beyond the muscularis mucosa layer. This results from an imbalance between mucosal protective factors (e.g. mucous production, bicarbonate secretion and blood flow) and mucosal aggressive factors (e.g. acid and pepsin). ${ }^{3}$ PUD is a major gastrointestinal-related reason for hospitalization. ${ }^{4}$ A reduction of acid output (vagotomy) is an integral part of surgical treatment of intractable ulcer and complications of this disease. However, the landmark discovery in 1983 of the role of Helicobacter pylori as a risk factor for PUD and its complications has

\author{
Corresponding author: \\ Emeka Ray-Offor \\ Department of Surgery, \\ University of Port Harcourt Teaching Hospital \\ Port Harcourt, Rivers State, \\ Nigeria. \\ Tel: +2348052077285 \\ Email: erayoffor@yahoo.com
}

tilted the management in favour of medical therapy. ${ }^{5}$

The prevalence of $H$. pylori infection is reported to be higher in developing countries especially in Africa, Central America, Central Asia, and Eastern Europe. ${ }^{6}$ The availability of effective medical eradication therapy for H. pylori and trends in use of over-the counter $\mathrm{H}_{2}$ antagonists, proton pump inhibitors and improved hygiene led to a decline in hospital admission from PUD. ${ }^{7}$ Other factors associated with increased risk of PUD are blood group $\mathrm{O}$, smoking and non-steroidal anti-inflammatory drugs. The clinical features of peptic ulcer may include epigastric pain related to meals, gastroesophageal reflux, dyspepsia and melena. In addition to gastrointestinal bleeding, complications include perforation, gastrointestinal obstruction and malignant transformation.

Endoscopy of the upper gastrointestinal tract with biopsy is the gold standard to differentiate between PUD and other causes of dyspepsia. ${ }^{8}$ Endoscopic therapies are effective at stopping bleeding and reducing the rates of referral to surgery and mortality. The interventions for haemostasis include injection therapy, thermal coagulation and mechanical methods with endoscopic suturing the more recent addition. ${ }^{9}$ Endoscopic balloon dilatation is effective in gastric outlet obstruction with short- and long-term remission. ${ }^{10}$ There is however 
paucity of data on non-perforated PUD from Niger delta region of Nigeria.

This study aimed to assess the current status and risk factors of peptic ulcer disease in a Nigerian metropolis.

\section{Patients and method}

A cross-sectional retrospective study of symptomatic patients diagnosed at a private referral endoscopy facility with peptic ulcer disease from February 2014 to September 2019. The endoscopy facility in the government tertiary hospital was non-functional during all but a few months of the entire study period.

The study was conducted in Port Harcourt metropolis, Niger delta region of Nigeria. The inclusion criteria were all cases with endoscopic diagnosis of deep mucosal breach of the stomach or duodenum. Exclusion criteria were paediatric patients (below 18 years of age), cases of ulcerated gastric tumour and superficial erosive gastritis. The variables studied included demographics, symptoms and duration, blood group, chronic non-steroidal anti-inflammatory use, smoking, endoscopic and histology findings.

The endoscopy equipment was Karl Storz (Germany) video gastroscopes 13821/13901PKS, camera unit, 100W Xenon light source/pump and high definition monitor.

A systematic examination was performed at upper GI endoscopy. Specific note of site, number and ulcer features was made, and tissue biopsy taken from non-bleeding ulcers. Endoscopic intervention, if any performed, was also noted. The risk of rebleed of ulcer was assessed using the Forrest classification. ${ }^{11}$ An active bleeding was recorded as Forrest 1 with active spurting vessel haemorrhage (A) or oozing haemorrhage (B). An evidence of recent haemorrhage was reported as nonbleeding visible vessel -IIA, adherent blood clot- IIB and haematin on ulcer base as IIC. Finally, lesions without active bleeding with clean base ulcers as Forrest III.

The tissue sample was fixed and transported in neutral $10 \%$ buffered formalin. Following paraffin embedding of sample tissue, microtome serial sections, staining with hematoxylin/eosin and Giemsa stains in a histopathology laboratory, the tissue slides were examined with light microscope by a pathologist. H. pylori was identified specifically on Giemsa-stained sections as light blue to grayish colored short rods within the luminal mucin or epithelial crypt.

Data analysis was performed using IBM SPSS Statistics for Windows, Version 20.0. Armonk, NY, USA. Age was shown as mean \pm standard deviation $(\mathrm{SD})$; number and percentage of cases were used for nominal and ordinal data. Categorical variables were compared using Pearson's Chi-square. $\mathrm{P}<0.05$ was considered statistically significant. A comparison of mean age of ulcer groups was performed by independent $t$ test.

\section{Results}

A total of 434 upper GI endoscopies were performed during the study period with thirty-one cases diagnosed with peptic ulcer disease. The yearly trend was an average of 6.7 cases/ year (figure 1). The age range of PUD patients was from 20 years to 74yrs (mean $53.3 \pm 18.5$ years). Males $16(51.6 \%)$ were marginally more affected than females 15(43.4\%)-Table 1. Comparatively, there was no statistical difference between the mean age of GU and DU cases- $54.4 \pm 20.2$ years and $48.1 \pm 14.5$ years respectively ( $p$ value 0.367 ) and no predilection for sex- Table 2. 


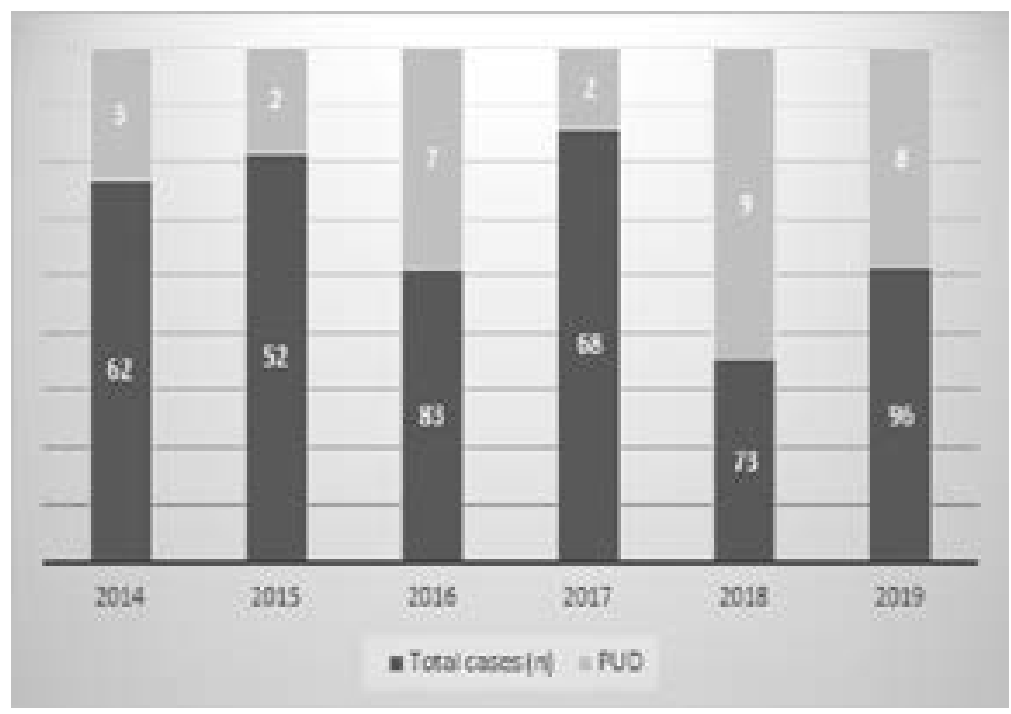

Figure 1. Trend of peptic ulcer disease cases from February 2014- September 2019

\begin{tabular}{|lc|}
\hline Variables & Frequency \\
Age(years) & \\
$<20$ & $0(0)$ \\
$20-44$ & $12(38.7)$ \\
$45-65$ & $10(32.3)$ \\
$>65$ & $9(29.0)$ \\
Sex & \\
Males & $16(51.6)$ \\
Females & $15(48.4)$ \\
Blood group & \\
O & $18(72.0)$ \\
A & $4(16.0)$ \\
B & $2(8.0)$ \\
AB & $1(4.0)$ \\
Not specified & 6 \\
Duration of symptoms & \\
$<1$ week & $14(45.2)$ \\
1-2weeks & $8(25.8)$ \\
$>$ 2weeks & $9(29.0)$ \\
Chronic NSAlD use & \\
Yes & $8(25.8)$ \\
No & $23(74.2)$ \\
Tobacco use & \\
Yes & $1(3.2)$ \\
No & $30(96.8)$ \\
\hline
\end{tabular}

*Two cases of coexisting gastric and duodenal ulcers excluded for clarity

\begin{tabular}{|lccc|}
\hline Variables & Gastric ulcer* & \multicolumn{2}{c|}{ Duodenal ulcer* p value } \\
Age (mean) & $54.4 \pm 20.2 \mathrm{yrs}$ & $48.1 \pm 14.5 \mathrm{yrs}$ & 0.367 \\
Sex & & & \\
$\quad$ Male & 8 & 7 & 0.550 \\
Female & 9 & 5 & \\
\hline
\end{tabular}


Blood group $\mathrm{O}$ was predominantly seen in $18(72.0 \%)$ of reported cases; however, a significant number of patients, not knowing their blood group had no record in their chart. A positive history of chronic use of non-steroidal anti-inflammatory drugs was seen in a quarter of cases-Table 1 . A history of smoking was documented in a sole case.

Gastrointestinal bleeding was the leading presenting complaint, 21(67.7\%), manifesting with melena in $17(54.8 \%)$ cases and haematemesis in $12(38.7 \%)$ cases. Next to this symptom was epigastric pain recorded in $13(41.9 \%)$. A delay of $>24 \mathrm{hrs}$ to endoscopy was recorded in all cases however two-fifth of cases (Table 1)

had their endoscopy within the first week of onset of symptoms.

Gastric ulcers were marginally more predominant than duodenal ulcers in a ratio of 1.4:1-Table 3. Solitary ulcers were predominant $-25(80.7 \%)$; however, multiple gastric ulcers were seen in 6 cases. Two patients were diagnosed with gastric and duodenal ulcers occurring together. The ulcer size and Forrest classification pattern are as shown in Table 3. Thirty-three (84.6\%) ulcers had clean base ulcers with no active bleeding. Seven cases $(22.5 \%)$ of tissue biopsy taken from non-bleeding ulcers were $H$. pylori positive from mucosal biopsy. There was no record of neoplastic transformation in any ulcer biopsied.

\begin{tabular}{|lc|}
\hline Ulcer variables & Frequency (\%) \\
Site & \\
Gastric ulcer & $17(54.8)$ \\
Duodenal ulcer & $12(38.7)$ \\
Gastric/Duodenal ulcers & $2(6.5)$ \\
& \\
Number per case & \\
One & $25(80.7)$ \\
Two & $4(12.9)$ \\
Three & $1(3.2)$ \\
Four & $1(3.2)$ \\
& \\
Size & \\
$<1 \mathrm{~cm}$ & $19(48.7)$ \\
1-2cm & $13(33.3)$ \\
$>2 \mathrm{~cm}$ & $7(18.0)$ \\
Forrest Classification* & \\
1A & $0(0)$ \\
IB & $1(2.6)$ \\
IIA & $1(2.6)$ \\
IIB & $3(7.6)$ \\
IIC & $1(2.6)$ \\
III & $33(84.6)$ \\
\hline
\end{tabular}

*Each ulcer detected assessed for size and graded.

\section{Discussion}

PUD is a gastrointestinal disorder of global importance. This study from a metropolitan South-South Nigeria population recorded an overall yearly diagnostic rate of $6.7 \%$. This overall rate is in line with the global prevalence trend of $5-10 \%$ for PUD. ${ }^{2}$ Despite the declining global trend of PUD there is age adjusted variability in prevalence. From this study $38.7 \%$ of population were below 45 years and no statistical predilection for sex noted in GU and DU but comprehensively males were marginally more affected. In Iran, among individuals aged 40-45 years alone, an overall prevalence of $41 \%$ for PUD is reported with males more affected than females. ${ }^{15}$ The mean age of gastric and duodenal ulcer patients also showed no statistical difference between the two groups. The reason for this age distribution may be that majority of gastrointestinal endoscopy cases were performed in patients above 45 years. A decline in duodenal ulcer and an unexpected rise in gastric ulcer has been reported in South-East and South- West Nige- 
ria. ${ }^{12,13}$ There is paucity of earlier documented literature from Niger delta Nigeria for comparison. In US, there is report of an age adjusted decline in hospitalization for PUD within the past four decades. ${ }^{14}$

Helicobacter pylori infection and non-steroidal anti- inflammatory drugs (NSAIDs) are two major factors impairing the mucosal resistance to result to injury. A low H. pylori infection rate $(21.4 \%)$ was recorded as bleeding ulcers were not biopsied so that further bleeding was not provoked. Approximately two third of the world's population is infected with Helicobacter pylori with the highest prevalence reported to be in Africa ${ }^{16} \mathrm{~A}$ previous study by one of the authors (ER-O), also showed a lower than expected $45 \%$ prevalence of $H$. pylori infection in a symptomatic cohort in this metropolitan population with possible reasons being ready access to over the counter acid reducing medications and practice of empirical eradication therapy for dyspepsia. ${ }^{17}$ This spiral, motile gram-negative organism is found in the mucin layer and fastens to gastric epithelium. The bacteria specifically attaches to phospholipids such as phosphatidyl ethanolamine, sialylated glycoproteins such as ganglioside monosialic 3(GM3), and Lewis B antigens present in persons with blood group $\mathrm{O} .{ }^{18} \mathrm{In}$ keeping with this, more than half of PUD cases in the study $(72.0 \%)$ were blood group O patients. A chronic use of NSAIDs and smoking were not frequent observations. Hence, a multifactorial etiology of peptic ulcer disease with an interplay of many factors such as the acid attack and the mucosal defense modulated by gender, blood group, age, $H$. pylori and various individual physiologic and probably genetic considerations. NSAIDs exert its ulcerogenic effect primarily because of inhibition of COX-1 thereby reducing mucosal generation of protective prostaglandins such as PGE2. ${ }^{19} \mathrm{~A}$ detailed alcohol intake history was not accessible from the patients records.

Gastric ulcers were more predominant than duodenal ulcers. Also notable was significant rate of multiple ulcers seen in one fifth of cases. The gastric antrum (prepyloric area) was the most affected site followed by the bulb of the duodenum. Duodenal ulcer can occur if acid secretion is enough to chronically lower the $\mathrm{pH}$ in the duodenal bulb and $H$. pylori infects and inflames the metaplastic or heterotopic gastric mucosa within the duodenal bulb. ${ }^{19}$ Clean base ulcers were seen in $84.6 \%$ of PUD cases despite a $67.7 \%$ frequency of gastrointestinal bleeding as presenting complaint. Thus, the rate of endoscopic intervention was low. This trend of non-bleeding ulcers was probably due to treatment by physicians before referral as $57.2 \%$ of cases underwent endoscopy after the first week of symptom onset. A sole case of endoscopic balloon dilatation for gastric outlet obstruction following an antral ulcer, an injection sclerotherapy and an endoscopic clip application were the three interventional procedures performed.

There were some limitations encountered in the course of this study. The sample size was small from this referral endoscopy facility, a strong reflection of the utilization rate of endoscopy in the locality. Another limitation was the sample population of mostly symptomatic patients which excludes asymptomatic ulcer cases. It will be a challenge however to do a population-based endoscopy study of asymptomatic patients in this low-middle income study setting from routine endoscopy for health check-up. Lastly, in the endoscopy-based setting of this study, perforated peptic ulcer disease was perfunctorily excluded.

\section{Conclusion}

There is a low diagnostic rate of peptic ulcer disease. Antral gastric ulcers are more commonly seen with gastrointestinal bleeding as the leading presenting symptom at endoscopy. A significant rate of $\mathrm{H}$. pylori-negative, NSAID-negative idiopathic ulcers is suggestive of a multifactorial aetiology. A multi-centre study is recommended for a broader view of the trend of PUD in the metropolis.

\section{Acknowledgement}

We wish to thank Prof NJ Jebbin for patiently reading through the manuscript and his useful suggestions.

\section{Conflict of interest}

None declared.

\section{References}

1. Moynihan BGA. History. Peptic ulcer. Philadelphia: WB Saunders, 1910: 17-23

2. Lanas A, Chan FKL. Peptic ulcer disease. Lancet 2017; 390(10094): 613-624.

3. Decross AJ, Marshall BJ. The role of Helicobacter pylori in acid peptic disease. Am J. Med Sci 1993; 306(6):381-92.

4. Sandler RS, Everhart JE, Donowitz M, Adams E, Cronin K, Goodman C, et al. The burden of selected digestive diseases in the United States. Gastroenterology. 2002; 122(5):1500-11.

5. Marshall BJ, Warren JR. Unidentified curved 
bacilli in the stomach of patients with gastritis and peptic ulceration. Lancet 1984; 1(8390):1311-5.

6. Hooi JKY, Lai WY, Ng WK, Suen MMY, Underwood FE, Tanyingoh D, et al. Global prevalence of Helicobacter pylori Infection: systematic review and meta-analysis. Gastroenterology 2017; 153(2): 420-429.

7. Sonnenberg A. Review article: historic changes of helicobacter pylori-associated diseases. Aliment. Pharmacol. Ther. 2013; 38(4): 329-342.

8. Agréus L, Talley NJ. Dyspepsia: current understanding and management. Annu Rev Med 1998; 49:47593 PubMed .

9. Agarwal A, Benias P, Brewer Gutierrez OI, Wong V, Hanada Y, Yang J, et al. Endoscopic suturing for management of peptic ulcer-related upper gastrointestinal bleeding: a preliminary experience. Endoscopy International Open 2018; 6(12): E1439-E1444.

10. Hamzaoui L, Bouassida M, Ben Mansour I, Medhioub M, Ezzine $\mathrm{H}$, Touinsi $\mathrm{H}$, et al. Balloon dilatation in patients with gastric outlet obstruction related to peptic ulcer disease. Arab J Gastroenterol 2015; 16(34):121-4.

11. Heldwein W, Schreiner J, Pedrazzoli J, Lehnert P. Is the Forrest classification a useful tool for planning endoscopic therapy of bleeding peptic ulcer? Endoscopy 1989; 21(6):258-62.

12. Nwokediuko SC, Ijoma U, Obienu O, Picardo
$\mathrm{N}$. Time trends of upper gastrointestinal diseases in $\mathrm{Ni}-$ geria. Ann Gastroenterol 2012; 25(1):52-56.

13. Ijarotimi O, Soyoye DO, Adekanle O, Ndububa DA, Umoru BI, Alatise OI. Declining prevalence of duodenal ulcer at endoscopy in Ile-Ife, Nigeria. $S A M J$ 2017; 107(9):750-3.

14. Feinstein LB, Holman RC, Yorita Christensen KL, Steiner CA, Swerdlow DL. Trends in hospitalizations for peptic ulcer disease, United States, 1998-2005. Emerg Infect Dis 2010; 16(9): 1410-8.

15. Sayehmiri K, Abangah G, Kalvandi G, Tavan H, Aazamir S. Prevalence of peptic ulcer in Iran: Systematic review and meta-analysis methods. J. Res Med Sci. 2018; $23: 8$.

16. Smith S, Fowora M, Pellicano R. Infections with Helicobacter pylori and challenges encountered in Africa. World J Gastroenterol 2019; 25(25): 3183-95.

17. Ray-Offor E, Obiorah CC. Helicobacter pylori and precancerous lesions of the stomach in a Nigerian Metropolis: A Cohort Study. Niger J Clin Pract 2018; 21(3):375-9.

18. Gold BD, Huesca M, Sherman PM, Lingwood CA. Helicobacter mustelae and Helicobacter pylori bind to common lipid receptors in vitro," Infection and Immunity 1993; 61(6):.2632-8.

19. Shiotani A, Graham DY. Pathogenesis and therapy of gastric and duodenal ulcer disease. Med Clin North Am 2002; 86(6): 1447-66. 